\title{
Comparison between solutions of the general dynamic equation and the kinetic equation for nucleation and droplet growth
}

Citation for published version (APA):

Holten, V., \& Dongen, van, M. E. H. (2009). Comparison between solutions of the general dynamic equation and the kinetic equation for nucleation and droplet growth. Journal of Chemical Physics, 130(1), 014102-1/8. [014102]. https://doi.org/10.1063/1.3054634

DOI:

$10.1063 / 1.3054634$

Document status and date:

Published: 01/01/2009

Document Version:

Publisher's PDF, also known as Version of Record (includes final page, issue and volume numbers)

Please check the document version of this publication:

- A submitted manuscript is the version of the article upon submission and before peer-review. There can be important differences between the submitted version and the official published version of record. People interested in the research are advised to contact the author for the final version of the publication, or visit the $\mathrm{DOI}$ to the publisher's website.

- The final author version and the galley proof are versions of the publication after peer review.

- The final published version features the final layout of the paper including the volume, issue and page numbers.

Link to publication

\footnotetext{
General rights

- You may freely distribute the URL identifying the publication in the public portal. follow below link for the End User Agreement:

www.tue.nl/taverne

Take down policy

If you believe that this document breaches copyright please contact us at:

openaccess@tue.nl

providing details and we will investigate your claim.
}

Copyright and moral rights for the publications made accessible in the public portal are retained by the authors and/or other copyright owners and it is a condition of accessing publications that users recognise and abide by the legal requirements associated with these rights.

- Users may download and print one copy of any publication from the public portal for the purpose of private study or research.

- You may not further distribute the material or use it for any profit-making activity or commercial gain

If the publication is distributed under the terms of Article 25fa of the Dutch Copyright Act, indicated by the "Taverne" license above, please 


\title{
Comparison between solutions of the general dynamic equation and the kinetic equation for nucleation and droplet growth
}

\author{
V. Holten ${ }^{\text {a) }}$ and M. E. H. van Dongen \\ Department of Applied Physics, Eindhoven University of Technology, P.O. Box 513, \\ 5600 MB Eindhoven, The Netherlands
}

(Received 17 October 2008; accepted 2 December 2008; published online 6 January 2009)

\begin{abstract}
A comparison is made between two models of homogeneous nucleation and droplet growth. The first is a kinetic model yielding the master equations for the concentrations of molecular clusters. Such a model does not make an explicit distinction between nucleation and droplet growth. The second model treats nucleation and growth separately, fully ignoring stochastic effects, and leads to the continuous general dynamic equation (GDE). Problems in applying the GDE model are discussed. A numerical solution of the kinetic equation is compared with an analytic solution of the GDE for two different cases: (1) the onset of nucleation and (2) the nucleation pulse. The kinetic model yields the thickness of the condensation front in size space as a function of supersaturation and dimensionless surface tension. If the GDE is applied properly, solutions of the GDE and the kinetic equation agree, with the exception of very small clusters, near-critical clusters, and the condensation front. () 2009 American Institute of Physics. [DOI: 10.1063/1.3054634]
\end{abstract}

\section{INTRODUCTION}

Condensation models play an important role in the simulation of devices such as steam turbines, gas-vapor separators, and combustion engines. At the heart of a condensation model lies the general dynamic equation (GDE) ${ }^{1-3}$ which describes the evolution of the droplet size distribution.

Usually, the GDE consists of independent nucleation and growth terms (and possibly coagulation terms, which are not considered here). The nucleation term describes nucleation as the addition of new droplets to the system at the smallest size considered, generally the critical size. A separate droplet growth model describes the evolution of the droplet population.

However, in reality, a single process - the stochastic size changes of molecular clusters-is responsible for both formation and growth. The kinetic model, based on this notion, consists of the kinetic master equations, which describe the concentration of clusters with discrete sizes. This model can be regarded as the most rigorous representation of a system with nucleation and has been in use $\mathrm{s}^{4,5}$ since the groundbreaking work of Courtney ${ }^{6}$ and Abraham. ${ }^{7}$

The GDE differs from the kinetic equation in three ways. First, in the GDE new droplets are inserted into the system at the critical size. There is no information on concentrations of droplets smaller than the critical size. Second, growth in the GDE is fully deterministic; stochastic effects are ignored. Third, the size distribution in the GDE is continuous, whereas in the kinetic equation it is discrete.

At present, little is known about the effects of the GDE approximations on the predicted droplet distributions. Gelbard and Seinfeld ${ }^{8}$ compared a discrete-continuous GDE with a fully continuous GDE and reported agreement. However, the boundary condition of the continuous GDE was

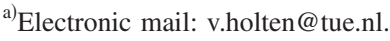

chosen such that it matched the solution of the discretecontinuous GDE. Chesnokov and Krasnoperov ${ }^{5}$ recently compared an extensive kinetic model with a more limited kinetic model and did not make a comparison with a continuous GDE.

Hagmeijer et al. ${ }^{9}$ applied the GDE to a condensing flow through a nozzle. In this study, the GDE was also used for droplets smaller than critical, while new droplets were still inserted at the critical size. It is unclear how accurate the GDE is below the critical size.

In this paper, we compare the GDE and the kinetic equation, both theoretically and numerically. The kinetic model is summarized and its relationship with the Fokker-Planck equation is shown. From that equation, the GDE is derived and an analytical solution of the GDE is given in the case of constant temperature and supersaturation. We then numerically evaluate the kinetic equation and the GDE in two test cases, focusing on the differences at both small and large droplet sizes.

\section{THEORY}

For the evaluation of the models, we consider a supersaturated vapor at temperature $T$ with monomer number density $\rho_{1}$. Both quantities are taken constant for simplicity. This approximation is reasonable when a dense carrier gas is present (for thermal equilibration) and the amount of molecules in droplets is much smaller than the amount of monomers.

The supersaturation $S$ is defined as $S \equiv \rho_{1} / \rho_{1}^{\text {sat }}$, with $\rho_{1}^{\text {sat }}$ the monomer density of a saturated vapor. The work of formation $W_{n}$ of a cluster with $n$ molecules is ${ }^{10}$

$$
W_{n} / k T=-n \ln S+\Theta n^{2 / 3}
$$

with $k$ Boltzmann's constant, and $\Theta \equiv\left(a_{1} \sigma\right) /(k T)$ with $\sigma$ the surface tension, $a_{1}=(36 \pi)^{1 / 3} v_{1}^{2 / 3}$ the molecular surface area, 
and $v_{1}$ the molecular volume. The work of formation reaches its maximum value at the critical size $n^{*}=[2 \Theta /(3 \ln S)]^{3}$.

\section{A. Kinetic model}

The kinetic model used here is the Szilard model, on which the derivation of classical nucleation theory is based. In this model, clusters can gain or lose only single molecules, and cluster-cluster interactions are neglected. The rate of change in the $n$-cluster concentration is then ${ }^{10,11}$

$$
\frac{\mathrm{d} f_{n}}{\mathrm{~d} t}=C_{n-1} f_{n-1}-\left(C_{n}+E_{n}\right) f_{n}+E_{n+1} f_{n+1}=J_{n-1}-J_{n},
$$

where $f_{n}$ is the number density of clusters with $n$ molecules, $C_{n}$ is the rate at which molecules condense on an $n$-cluster, and $E_{n}$ is the rate at which molecules evaporate from such a cluster. The current or flux $J_{n}$, the net number of clusters per unit time and volume that grow from size $n$ to $n+1$, is ${ }^{10}$

$$
J_{n} \equiv C_{n} f_{n}-E_{n+1} f_{n+1} .
$$

The condensation coefficient is the product of the sticking probability (assumed to be unity), the collision frequency per unit area, and the cluster surface area, ${ }^{10}$

$$
C_{n}=\rho_{1}\left(\frac{k T}{2 \pi m}\right)^{1 / 2} \times a_{1} n^{2 / 3},
$$

where $m$ is the mass of a molecule. The evaporation coefficient $E_{n}$ is found by the detailed balance equation: at saturation ( $S=1$, denoted by superscript "sat") all $J_{n}$ equal zero, so Eq. (3) becomes

$$
E_{n+1}=C_{n}^{\text {sat }}\left(f_{n}^{\text {sat }} / f_{n+1}^{\text {sat }}\right) \text {. }
$$

The evaporation coefficient is assumed to be supersaturation independent. For the size distribution at saturation $f_{n}^{\text {sat }}$, we take the Courtney form, ${ }^{12,13}$

$$
f_{n}^{\text {sat }}=\rho_{1}^{\text {sat }} \exp \left(-\Theta n^{2 / 3}\right), \quad n \geq 2 .
$$

The combination of Eqs. (5) and (6) allows writing $E_{n}$ as

$$
\begin{aligned}
E_{n} & =C_{n-1}^{\mathrm{sat}} \exp \left\{\Theta\left[n^{2 / 3}-(n-1)^{2 / 3}\right]\right\} \\
& \approx C_{n-1}^{\mathrm{sat}} \exp \left(\frac{2}{3} \Theta n^{-1 / 3}\right) .
\end{aligned}
$$

For constant temperature and supersaturation, all fluxes $J_{n}$ eventually converge to the same steady-state value denoted by $J$, given by ${ }^{14}$

$$
J=\left(\sum_{n=1}^{\infty} \frac{1}{C_{n} S^{n} f_{n}^{\mathrm{sat}}}\right)^{-1} .
$$

\section{B. Fokker-Planck equation}

The set of equations of Eq. (2) can be transformed into a single equation in which $n$ is a continuous variable. The quantities $f_{n}(t), C_{n}$, and $E_{n}$ become the functions $f(n, t)$, $C(n)$, and $E(n)$; the arguments $n$ and $t$ will usually be omitted. Following Kashchiev, ${ }^{11}$ quantities evaluated at $n-1$ and $n+1$ are approximated by second-order expansions around $n$,

$$
\begin{aligned}
& C_{n-1} f_{n-1} \approx C f-\frac{\partial}{\partial n}(C f)+\frac{1}{2} \frac{\partial^{2}}{\partial n^{2}}(C f), \\
& E_{n+1} f_{n+1} \approx E f+\frac{\partial}{\partial n}(E f)+\frac{1}{2} \frac{\partial^{2}}{\partial n^{2}}(E f) .
\end{aligned}
$$

Equation (2) then becomes a Fokker-Planck equation, ${ }^{15}$

$$
\frac{\partial f}{\partial t}=\frac{1}{2} \frac{\partial^{2}}{\partial n^{2}}[(C+E) f]-\frac{\partial}{\partial n}[(C-E) f] .
$$

The first term on the right-hand side of Eq. (12) corresponds to diffusion in $n$-space caused by stochastic fluctuations of the cluster size, with a diffusion coefficient of $\frac{1}{2}(C+E)$. The second term represents the deterministic cluster growth or shrinkage caused by the difference between condensation and evaporation, yielding a drift coefficient of $(C-E)$.

It is advantageous to replace the number of molecules $n$ in the cluster by its radius $r=r_{1} n^{1 / 3}$, where $r_{1}=\left(3 v_{1} / 4 \pi\right)^{1 / 3}$ is the molecular radius. The radius distribution function $F(r, t)$ replaces the earlier distribution $f(n, t)$, according to $f \mathrm{~d} n$ $=F \mathrm{~d} r$. The Fokker-Planck equation in $r$ space then becomes

$$
\frac{\partial F}{\partial t}=\frac{\partial}{\partial r}\left(D \frac{\partial F}{\partial r}\right)-\frac{\partial(\dot{r} F)}{\partial r},
$$

with diffusion coefficient

$$
D(r)=\frac{1}{2}\left(\frac{\mathrm{d} r}{\mathrm{~d} n}\right)^{2}(C+E) \approx \frac{r_{1}^{4}}{18 r^{2}} C_{1}^{\mathrm{sat}}\left[S+e^{K e(r)}\right]
$$

and growth rate

$$
\begin{aligned}
\dot{r}(r) & =\frac{\mathrm{d} r}{\mathrm{~d} n}(C-E)-\frac{1}{2} \frac{\mathrm{d} r}{\mathrm{~d} n} \frac{\partial}{\partial r}\left[(C+E) \frac{\mathrm{d} r}{\mathrm{~d} n}\right] \\
& \approx \frac{1}{3} r_{1} C_{1}^{\mathrm{sat}}\left[S-\left(1-\frac{\Theta}{9} \frac{r_{1}^{4}}{r^{4}}\right) e^{K e(r)}\right],
\end{aligned}
$$

where the kelvin number is defined by

$$
\operatorname{Ke}(r) \equiv \frac{2}{3} \Theta\left(r_{1} / r\right)=\left(r^{*} / r\right) \ln S .
$$

The approximate results of Eqs. (14) and (16) were obtained by using Eq. (8) and the approximation $C_{n-1}^{\text {sat }} \approx C_{n}^{\text {sat }}$. From here on, we also discard the term with $\left(r_{1} / r\right)^{4}$ in the expression for $\dot{r}$, leading to a simplified growth rate known as the Hertz-Knudsen growth law,

$$
\dot{r}(r)=\frac{1}{3} r_{1} C_{1}^{\mathrm{sat}}\left[S-e^{K e(r)}\right] .
$$

For large droplets, $K e \rightarrow 0$ and the growth rate $\dot{r}$ becomes radius independent. The $\mathrm{e}^{K e}$ term in Eq. (18) represents the Kelvin effect, which corrects for the droplet curvature. At the critical radius, $e^{K e}=S$ and the growth rate is zero. An important consequence is that at the critical radius the drift flux $\dot{r} F$ in Eq. (13) equals zero, so that the passage of the critical radius in size space is always dominated by diffusion.

\section{General dynamic equation}

The first step in deriving the GDE from the FokkerPlank equation is neglecting the diffusion term. This is justified because the diffusion coefficient $D$ of Eq. (14) rapidly 


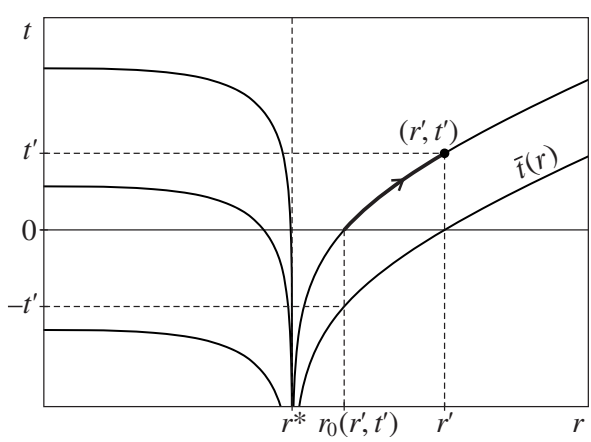

FIG. 1. Schematic $r$ - $t$ diagram with several characteristics of the general dynamic equation, $\partial F / \partial t=-\partial(\dot{r} F) / \partial r$. At the critical radius $r^{*}$, which is taken time independent, the growth rate is zero and the characteristics are asymptotically vertical. The dot is a droplet with radius $r^{\prime}$ at time $t^{\prime}$, with initial radius $r_{0}\left(r^{\prime}, t^{\prime}\right)$ and radius history shown by a thick line.

approaches zero for clusters with radii $r \gg r_{1}$. We shall ignore here the dominance of the diffusion term near the critical radius. The GDE thus becomes ${ }^{2,11}$

$$
\frac{\partial F}{\partial t}=-\frac{\partial(\dot{r} F)}{\partial r} .
$$

Equation (19) is sometimes called the "condensation equation." It can be solved by the method of characteristics, and for time-independent $\dot{r}$ the solution is ${ }^{16}$

$$
F(r, t)=\frac{1}{\dot{r}(r)} g[\bar{t}(r)-t],
$$

with

$$
\bar{t}(r) \equiv \int_{r_{\text {ref }}}^{r} \frac{1}{\dot{r}\left(r^{\prime}\right)} \mathrm{d} r^{\prime} .
$$

Here the lower integration limit $r_{\text {ref }}$ can be chosen arbitrarily, as long as $r^{*}$ is not in the integration interval (the integrand is singular there). The function $g(t)$ is determined by the initial conditions and the choice of $r_{\text {ref }}$, so that the function $F(r, t)$ is independent of $r_{\text {ref }}$.

The function $\bar{t}(r)$ of Eq. (21) describes the characteristic curve in the $r$ - $t$ plane that crosses the $t$ axis at $r=r_{\text {ref. }}$ Other characteristics can easily be found by shifting the curve in the $t$ direction, and Fig. 1 shows several of these. Later, we will also require the inverse function of $\bar{t}(r)$, denoted by $\bar{r}(t)$. The $\bar{r}(t)$ function gives the radius of the droplet formed at $t=0$ and $r=r_{\text {ref }}$ as a function of time.

The absence of the diffusion term means that the GDE of Eq. (19) cannot describe the nucleation process, the growth of clusters from monomers to the critical size. The growth rate $\dot{r}$ is negative for clusters that are smaller than the critical size, so that supercritical droplets cannot appear. To include nucleation in the GDE, usually a source term is added to Eq. (19) in the form of a delta function, ${ }^{2,17}$ so that the GDE becomes

$$
\frac{\partial F}{\partial t}=-\frac{\partial(\dot{r} F)}{\partial r}+\delta\left(r-r^{*}\right) J,
$$

where $J$ is the steady-state nucleation rate. The nucleated clusters are usually inserted at the critical size because a cluster of size $r^{*}$ is regarded as the smallest stable cluster. ${ }^{2}$ However, from a thermodynamical point of view, a critical cluster is in unstable equilibrium. Therefore, in most growth models, the growth rate of a critical cluster equals zero. The solution of Eq. (22) then becomes problematic because the nucleation term can lead to an unlimited droplet concentration at $r^{*}$. Still, Eq. (22) is commonly used. In those cases the problem at $r^{*}$ does not occur due to a time-dependent critical radius, ${ }^{9}$ for example.

If the critical radius is constant, the GDE therefore cannot be used with the above delta function. A straightforward way to avoid problems is to introduce nucleated droplets at a radius $r_{\mathrm{n}}$ that is slightly larger than $r^{*}$, thus forcing the growth rate of the droplets to be positive. Such a modification leads to a GDE in the form

$$
\frac{\partial F}{\partial t}=-\frac{\partial(\dot{r} F)}{\partial r}+\delta\left(r-r_{\mathrm{n}}\right) J, \quad r_{\mathrm{n}}>r^{*} .
$$

We will now solve this GDE with the initial condition

$$
F(r, 0)=F_{0}(r) \text {. }
$$

Hagmeijer ${ }^{17}$ presented the general solution of such a GDE in which $J, \dot{r}$, and $r^{*}$ may be time dependent. If these parameters are constant in time, as they are here, a solution can be obtained in a more straightforward way, as we will now show.

The general solution of the GDE is equal to the general solution of the homogeneous equation [Eq. (19)], with condition (24), plus a particular solution of Eq. (23) with condition $F(r, 0)=0$. We start with the homogeneous problem of Eq. (19), which represents the evolution of an existing distribution with negligible nucleation. The solution has already been given in a general form in Eq. (20). Evaluation at $t=0$ and substitution of Eq. (24) results in an expression for the function $g$,

$$
g[\bar{t}(r)]=F_{0}(r) \dot{r}(r) .
$$

For times $t \geq 0$ we can write Eq. (20) as

$$
F(r, t)=\frac{g[\bar{t}(r)-t]}{\dot{r}(r)}=\frac{g\left[\bar{t}\left(r_{0}\right)\right]}{\dot{r}(r)},
$$

with

$$
r_{0}(r, t) \equiv \vec{r}[\bar{t}(r)-t] .
$$

The function $r_{0}(r, t)$ gives the starting radius of a droplet, that is, the radius the droplet had at $t=0$. Using the value of $g$ from Eq. (25), the solution of the homogeneous problem becomes

$$
F(r, t)=F_{0}\left[r_{0}(r, t)\right] \frac{\dot{r}\left[r_{0}(r, t)\right]}{\dot{r}(r)},
$$

which corresponds to the solutions obtained by Loyalka and Park ${ }^{18}$ and Hagmeijer. ${ }^{17}$ The expression for $r_{0}$ in Eq. (27) can be simplified by choosing $r_{\text {ref }}=r$, resulting in $r_{0}(r, t)=\bar{r}(-t)$. This relation is illustrated in Fig. 1.

Next, we consider the particular solution of Eq. (23) with initial condition $F(r, 0)=0$. This problem corresponds to nucleation at constant temperature and supersaturation, with 
no droplets present initially. In this case, nucleation can also be represented in the homogeneous equation [Eq. (19)] by the condition of constant flux $F \dot{r}$ at the radius $r_{\mathrm{n}}{ }^{11}$

$$
F\left(r_{\mathrm{n}}, t\right) \dot{r}\left(r_{\mathrm{n}}\right)=J, \quad t>0 .
$$

This boundary condition replaces the delta function in Eq. (23). When substituted in Eq. (20), the initial and boundary conditions completely determine the function $g$; it is a step function, whose value changes from $J$ to 0 at an argument of $\bar{t}\left(r_{\mathrm{n}}\right)$. When $r_{\mathrm{ref}}=r_{\mathrm{n}}$ is chosen for simplicity, the solution for the distribution becomes

$$
F(r, t)= \begin{cases}J / \dot{r}(r), & r_{\mathrm{n}} \leq r<\bar{r}(t) \\ 0, & \text { otherwise },\end{cases}
$$

which is a special case of the solution presented by Kashchiev. ${ }^{11}$ The function $\bar{r}(t)$ with $r_{\text {ref }}=r_{\mathrm{n}}$ gives the radius history of the first droplet, the one formed at $r=r_{\mathrm{n}}$ and $t=0$. Since this droplet is the largest one at any moment in time, the function $\bar{r}(t)$ also represents the radius at the front of the distribution.

For our computations, we will not need the general solution of the inhomogeneous GDE of Eq. (23). It can, however, be easily obtained by adding the right-hand sides of Eqs. (28) and (30).

\section{NUMERICAL APPROACH}

\section{A. Kinetic model}

The system of differential equations in the kinetic model [Eq. (2)] was reduced by the discrete section method, ${ }^{19}$ where size space is divided into sections or bins. This method is most often used to solve large systems of kinetic differential equations and is accurate if the number of bins is large enough. ${ }^{5}$ When the division into bins is made, the average cluster concentration $f$ in a bin depends only on the fluxes $J_{n}$ at the bin edges. These fluxes are estimated using a linear interpolation of average $f$ between two adjacent bins. After sectioning, a reduced system is obtained with one differential equation for each bin. The structure of the system is the same as Eq. (2), but with different coefficients in the place of $C_{n}$ and $E_{n}$, and bin averages of $f$ instead of pure $f_{n}$. If a bin has unit length, its differential equation reduces to the original equation from Eq. (2).

Usually, the length of a bin is taken proportional to $n,{ }^{5,19,20}$ leading to a constant number of bins per order of magnitude of the cluster radius $r$. Instead, we chose the bin lengths such that the bin edges are equidistant in $r$ space, and the bin length is then approximately proportional to $n^{2 / 3}$. Our choice is better suited to the problem we are solving, for two reasons. First, we convert the computed distribution to $r$ space, and the density of data points along the $r$ axis is then constant. Second, because the growth rate $\dot{r}$ converges to a constant, the growth rate of the number of bins also becomes constant.

We used a bin length of $0.005 r_{1}$ in $r$ space. In $n$ space, the bin size is discrete and rounded to the nearest integer (or to 1 if it is less than 1). The first 1000 bins then have unit length. Comparisons were made with a bin length of
$0.0011 r_{1}$ (for which the first 10000 bins have unit length) and no significant differences in the cluster size distribution were found.

The equations were made dimensionless by scaling the cluster concentrations with the monomer concentration $\rho_{1}$ and by introducing a dimensionless time $\tau \equiv C_{1} t$. The reduced system was solved by numerical integration using the Crank-Nicolson method, ${ }^{21}$ with dimensionless time steps in the range of 0.02-0.04. The lower limit of the solution was taken at $n=2$ instead of $n=1$, by keeping the concentration of dimers at the constant value $f_{2}=\rho_{1}^{\text {sat }} \exp \left(-W_{2} / k T\right)$, consistent with the Courtney distribution. ${ }^{12}$ This limit was chosen because starting at $n=2$ allowed the use of larger time steps, while the solution is relatively insensitive to the lower limit. As a test, several solutions with lower limits at $n=1$ and $n$ $=2$ were compared, and negligible difference was found.

\section{B. General dynamic equation}

The GDE was made dimensionless in the same way as the kinetic equations. In addition, the $r$ coordinate was made dimensionless by scaling it with $r_{1}$.

In the case of nucleation without an existing distribution, the solution was directly computed from Eq. (30). In the case of an existing distribution without nucleation, a different approach was taken. Before calculating the solution, the timeindependent values of the $\bar{t}(r)$ function were computed in the size range of interest, as follows. The $r$ range was divided into the parts below and above $r^{*}$. Different fixed values of $r_{\text {ref }}$ were taken for each part, namely, $r_{\text {ref }}=0$ for $r<r^{*}$ and the arbitrary value of $r_{\text {ref }}=1.001 r^{*}$ for $r>r^{*}$. To maintain accuracy in the range around $r^{*}$, where $\bar{t}(r)$ is very steep, a coordinate transformation was applied, which made $\bar{t}$ an approximately linear function of the transformed coordinate. Equation (21) was then used to compute and store a table of values of the $\bar{t}$ function. Interpolation between table values yielded $\bar{t}$ values with a relative accuracy better than $10^{-4}$. The $\bar{r}(t)$ function was not precomputed but implemented as the numerical solution of $r$ in the equation $t=\bar{t}(r)$. Equations (27) and (28) were finally used to obtain the solution at several times using the same precomputed $\bar{t}(r)$ for each time.

\section{NUMERICAL COMPARISON OF THE MODELS}

\section{A. Transient nucleation at constant temperature and supersaturation}

Both the kinetic equation and the GDE were evaluated for conditions corresponding to water vapor at $228 \mathrm{~K}$ with a supersaturation of 20.6 (shown in Table I, line A0), with no clusters present initially. The resulting distributions are compared on a logarithmic scale in Fig. 2(a) and on a linear scale in Fig. 2(b). While the logarithmic plot shows the differences between the distributions well, a linear plot is necessary to assess the predicted total droplet concentrations (the area under the distribution).

A major difference is the absence of clusters below the critical size in the GDE result, in contrast to the high subcritical concentrations in the result from the kinetic model. 
TABLE I. Conditions of the numerical simulations. (All conditions represent water vapor. Liquid density and saturated vapor pressure were computed with correlations from Ref. 22, and the surface tension was taken from Ref. 23.)

\begin{tabular}{|c|c|c|c|c|c|c|c|c|c|}
\hline Condition $^{\mathrm{a}}$ & $\begin{array}{c}T \\
(\mathrm{~K})\end{array}$ & $\begin{array}{c}p \\
(\mathrm{~Pa})\end{array}$ & $S$ & $\Theta$ & $r^{*} / r_{1}$ & $\begin{array}{c}\rho_{1} \\
\left(\mathrm{~m}^{-3}\right)\end{array}$ & $\begin{array}{c}C_{1} \\
\left(\mathrm{~s}^{-1}\right)\end{array}$ & $\begin{array}{c}J \\
\left(\mathrm{~m}^{-3} \mathrm{~s}^{-1}\right)\end{array}$ & $\begin{array}{c}r_{1} \\
(\mathrm{~nm})\end{array}$ \\
\hline A0 & 228.0 & 225.0 & 20.57 & 12.74 & 2.81 & $7.148 \times 10^{22}$ & $4.406 \times 10^{6}$ & $2.849 \times 10^{13}$ & 0.195 \\
\hline A1 & 253.2 & 292.5 & 2.319 & 10.61 & 8.41 & $8.366 \times 10^{22}$ & $5.342 \times 10^{6}$ & $2.642 \times 10^{-80}$ & 0.193 \\
\hline B0 & 217.8 & 116.2 & 34.2 & 13.7 & 2.59 & $3.863 \times 10^{22}$ & $2.348 \times 10^{6}$ & $8.509 \times 10^{13}$ & 0.196 \\
\hline B1 & 226.7 & 128.4 & 13.5 & 12.9 & 3.29 & $4.102 \times 10^{22}$ & $2.524 \times 10^{6}$ & $3.390 \times 10^{7}$ & 0.195 \\
\hline $\mathrm{C} 0$ & 239.6 & 407.3 & 11.3 & 11.7 & 3.22 & $1.231 \times 10^{23}$ & $7.711 \times 10^{6}$ & $1.507 \times 10^{11}$ & 0.194 \\
\hline $\mathrm{C} 1$ & 244.3 & 427.7 & 7.55 & 11.3 & 3.73 & $1.268 \times 10^{23}$ & $7.992 \times 10^{6}$ & $1.491 \times 10^{6}$ & 0.194 \\
\hline
\end{tabular}

${ }^{\mathrm{a}}$ Numbers 0 and 1 denote conditions during and after the pulse, respectively, and $\mathrm{B}$ and $\mathrm{C}$ refer to the two cases in Fig. 6.

This is, of course, caused by the introduction of new droplets at $r_{\mathrm{n}}$ in the GDE. On the other hand, the GDE overpredicts the concentration of clusters that are slightly larger than critical because the growth rate is small there and the concentration is inversely proportional to it. The peak in the GDE result near $r^{*}$ thus reflects the singular behavior of the original GDE [Eq. (22)] at the critical size. In the distribution from the kinetic equation, however, there is no singularity or discontinuity at the critical size. On the contrary, the concentration decreases smoothly with increasing radius.

It is interesting to note that Ramabhadran et al $^{24}$ derived a condition for which the diffusion term in the FokkerPlanck equation can be neglected with respect to the drift term. They state that for most distributions this condition is satisfied and the simplification is justified. However, it is clear that for sizes near the critical size and in the front region, the diffusion term remains important.

It appears from Fig. 2 that the distribution eventually becomes independent of $r$ for large droplets. In fact, the steady-state distributions from both models are radius independent for large radii because the growth rate $\dot{r}$ is also radius independent there.

The front of the distribution is also a region with notable differences between the distributions. The front of the GDE distribution is always sharp, unlike the smooth front from the kinetic equation because there is no diffusion in the GDE.
However, diffusion is not the only cause of the flattening of the front in the kinetic model. For small radii, the growth rate is strongly size dependent (large droplets grow faster than small ones) and this stretches the front over a larger radius region as it moves in radius space. Another reason for the smooth front is the initial time dependence of the nucleation rate in the kinetic model, whereas the nucleation rate in the GDE is taken constant in time. Including the time dependence of the nucleation rate in the GDE, like in Ref. 11, will probably improve the agreement with the kinetic equation in the front region.

The thickness of the front from the kinetic equation depends on the nucleation conditions. To quantify the dependence, we define the front thickness $\Delta r$ as

$$
\Delta r \equiv \frac{F_{\mathrm{s}}\left(r_{\max }\right)}{|\partial F / \partial r|_{\max }},
$$

where $|\partial F / \partial r|_{\max }$ is the maximum of the absolute value of $\partial F / \partial r$ and $r_{\max }$ is the radius at which the maximum occurs. Further, $F_{\mathrm{s}}(r)=J / \dot{r}(r)$ is the steady-state radius distribution. $\left(F_{\mathrm{s}}\right.$ is the steady-state value from the GDE, but for $r \gg r^{*}$ the kinetic model has the same steady-state value.) The thickness increases as the distribution grows to larger radii but eventually becomes constant as the diffusion becomes negligible
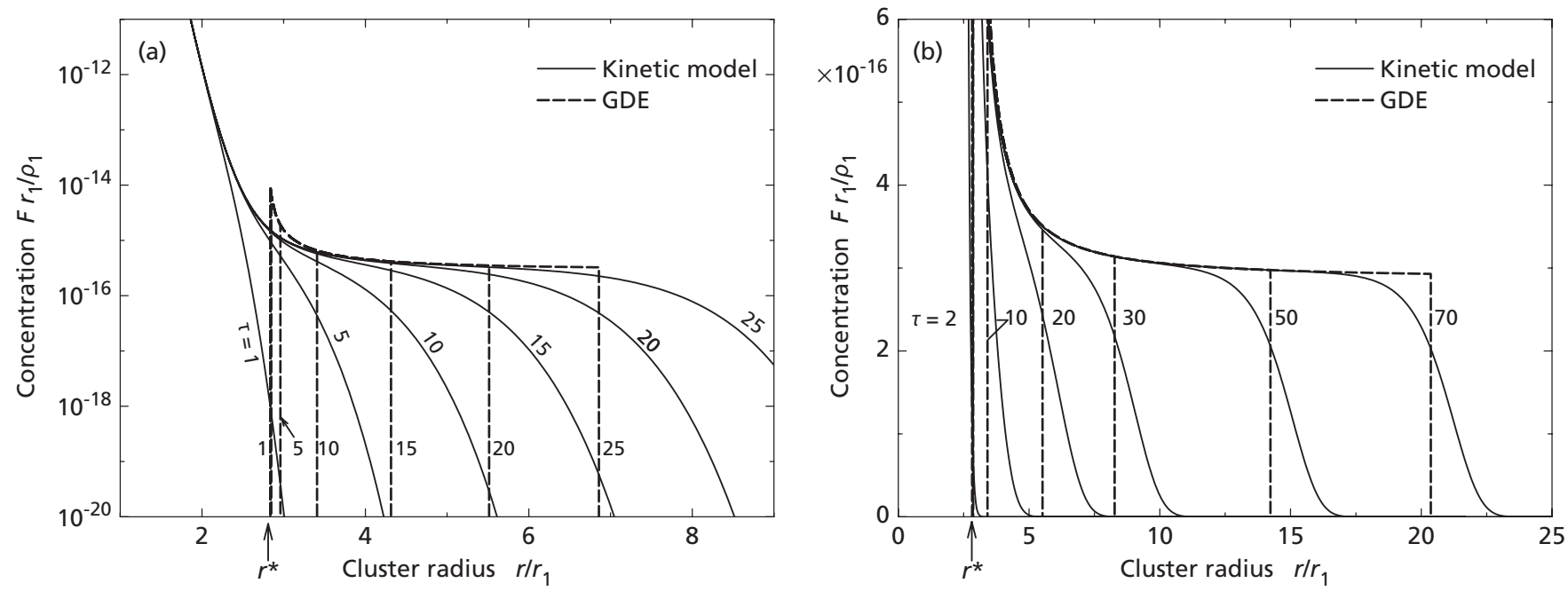

FIG. 2. Radius distribution function during nucleation, according to the kinetic equation and the GDE, on (a) a logarithmic scale and (b) a linear scale. Conditions are given in Table I, line A0. Distributions are shown for several dimensionless times $\tau=t C_{1}$. The GDE distributions at $\tau=1$ and $\tau=2$ are so narrow that the beginning is indistinguishable from the end. The initial radius in the GDE was taken equal to $r_{\mathrm{n}}=1.01 r^{*}$. 


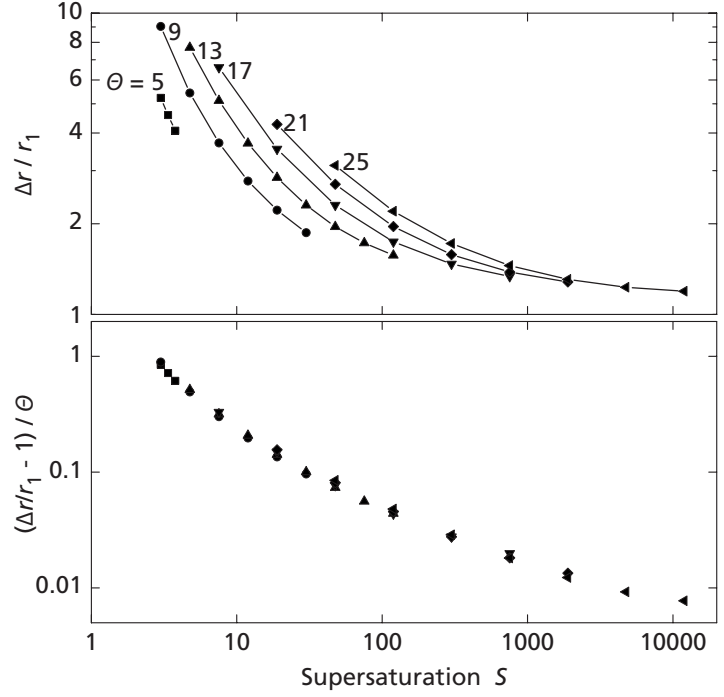

FIG. 3. Top: thickness $\Delta r$ of the front from the kinetic equation, scaled with $r_{1}$, as a function of the supersaturation. Bottom: subtracting unity and dividing by the dimensionless surface tension $\Theta$ collapse all curves onto a single curve.

and the growth rate becomes constant. The asymptotic thickness, therefore, depends only on $S$ and $\Theta$.

The asymptotic thickness of the front was calculated for $S \geq 3$ and $\Theta$ between 5 and 25 , while keeping the critical size $n^{*}$ above five molecules, and the dimensionless nucleation rate $J /\left(C_{1} \rho_{1}\right)$ between $10^{-100}$ and $10^{-5}$. Figure 3 demonstrates that the thickness is largest for low $S$ values and seems to converge to $r_{1}$ for large $S$. The value $\left(\Delta r / r_{1}\right)-1$ is approximately proportional to $\Theta$, as the lower part of Fig. 3 shows.

The GDE front position is to some extent arbitrary because it depends on $r_{\mathrm{n}}$, which is a free parameter. We can therefore choose a $r_{\mathrm{n}}$ value such that the GDE matches the kinetic equation best. Specifically, $r_{\mathrm{n}}$ can be chosen such that the number density of clusters in the front region is equal for both models. This condition implies that the integral $\int F(r) \mathrm{d} r$ over the front should be equal. We have computed the asymptotic value of $r_{\mathrm{n}}$ that satisfies this requirement, for the same $S$ and $\Theta$ values as above. As Fig. 4 displays, the $r_{\mathrm{n}}$ needs to be several percent higher than the critical size. A higher $r_{\mathrm{n}}$ is needed for higher $S$ and lower $\Theta$ values.

\section{B. Nucleation pulse}

An important experimental technique for determining nucleation rates is the nucleation pulse method..$^{22,23,25}$ In this method, a gas-vapor mixture is first rapidly expanded and then recompressed. The expansion causes a temperature drop and a consequent supersaturation increase. Nucleation takes place during the pulse, a short $(0.1-1 \mathrm{~ms})$ period between the expansion and recompression, when the pressure, temperature, and supersaturation are held constant. The recompression of the mixture reduces the supersaturation and stops nucleation. However, the supersaturation after the pulse remains larger than unity, so the droplets that were formed during the pulse grow until they can be detected.

We have evaluated both models in an idealized nucle-

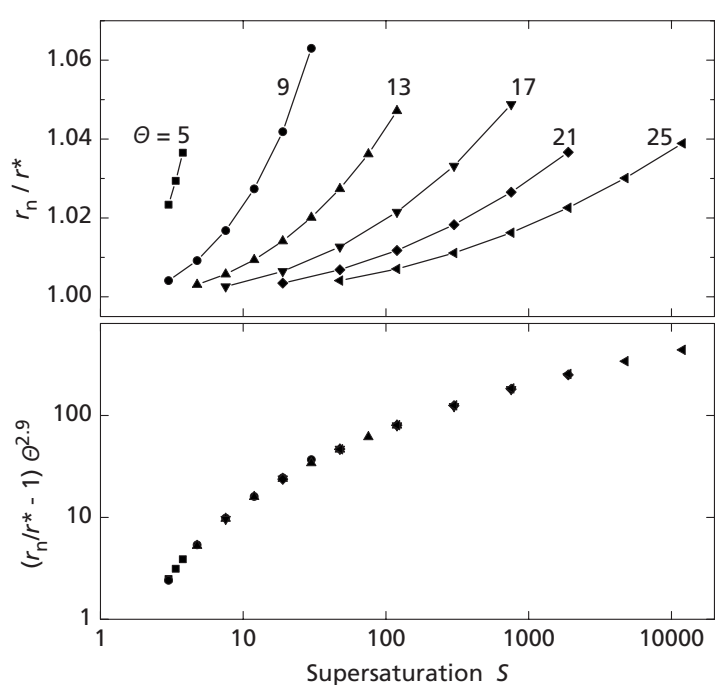

FIG. 4. Top: initial radius $r_{\mathrm{n}}$ in the GDE that is required to match the number of droplets predicted by the kinetic equation. Bottom: subtracting unity and multiplying by $\Theta^{2.9}$ collapse all curves onto a single curve.

ation pulse. The expansion stage was neglected, and the computation was started at the pulse conditions. The pulse stage then corresponds to the case of constant temperature and supersaturation that has already been covered in Sec. IV A. We also ignored the recompression time at the end of the pulse and reduced the supersaturation instantaneously. Furthermore, in the GDE we neglected the small nucleation rate after the pulse, so that the homogeneous solution [Eq. (28)] applies.

Figure 5 shows the evolution of the distribution after a pulse of dimensionless duration $C_{1} \Delta t=220$. This corresponds to a duration of $0.05 \mathrm{~ms}$, which is quite short and has a pronounced effect on the shape of the distribution function. The pulse conditions are taken the same as the conditions of Fig. 2, and the conditions after the pulse represent water vapor at $253 \mathrm{~K}$ with a supersaturation of 2.3 (Table I, line A1).

The increase in $r^{*}$ causes a rapid collapse of the distribution function at small sizes. In the deterministic GDE, all droplets that become subcritical after the pulse (droplets sized between $r_{0}^{*}$ and $r_{1}^{*}$ in Fig. 5) will eventually evaporate. The sharp lower boundary of the GDE distribution moves to smaller sizes (at $\tau=0.45$ it can be seen as a vertical straight line) until it reaches $r=0$ and the part of the GDE distribution below $r_{0}^{*}$ becomes smooth. The kinetic distribution shows a similar collapse but does not decrease toward zero at small sizes. Instead, the distribution converges to the new steadystate distribution, which is lower than the old one, but still high at small sizes. Interestingly, the GDE prediction is accurate down to about $r=2 r_{1}$, which is an even smaller radius than the original minimum radius of the distribution near $r_{0}^{*}$.

As before, the two models also differ in the front region. In our example, the change in conditions at the end of the pulse does not have an effect on the thickness of the front because the front is located at radii much larger than the critical size. Only the propagation speed of the front decreases.

At intermediate droplet sizes, the agreement between 

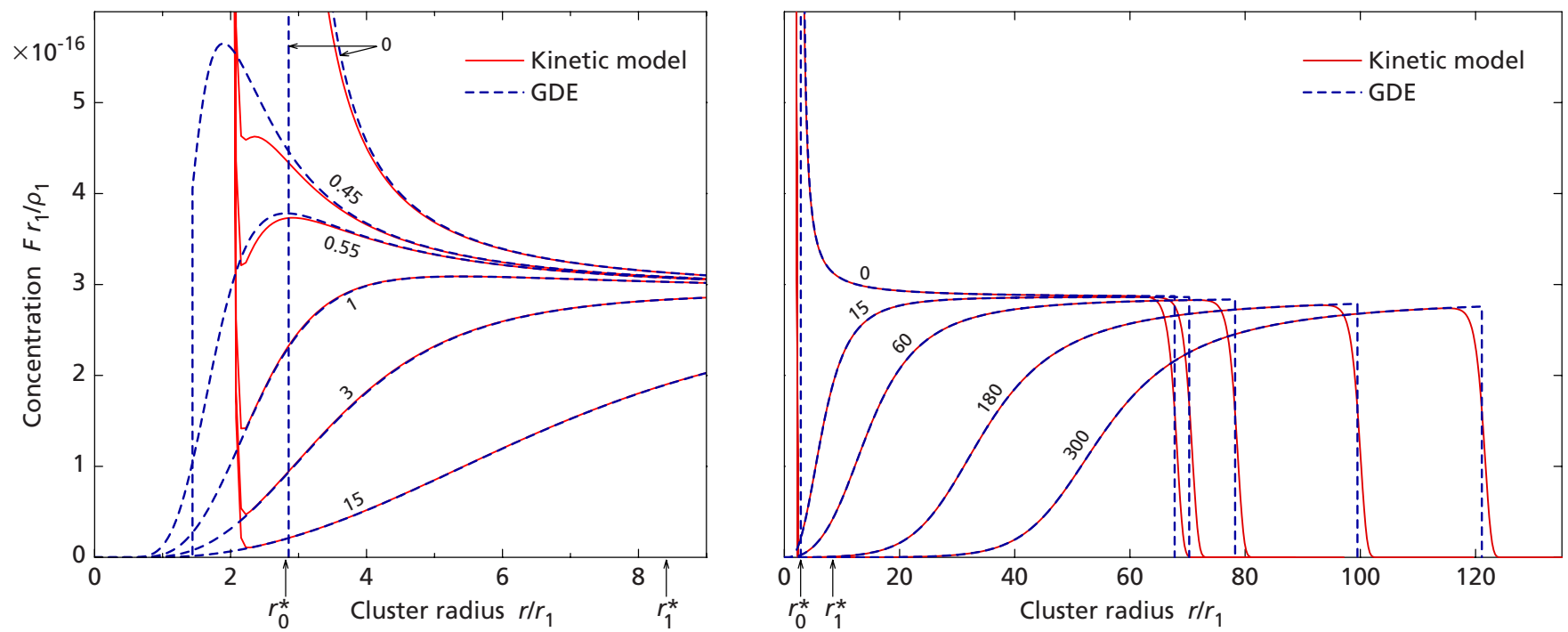

FIG. 5. (Color online) Radius distribution function at the end of and after the nucleation pulse, according to the kinetic equation and the GDE. The left part of the figure is a magnification of the distributions at small sizes. Numbers near the curves are the dimensionless times $\tau=t C_{1}$ since the end of the pulse. The value of the critical size during the pulse $r_{0}^{*}$ and after the pulse $r_{1}^{*}$ is indicated. Near $r_{0}^{*}$, the dashed vertical line is the minimum radius of the GDE distribution at the end of the pulse. The solid near-vertical curves near $r / r_{1}=2$ approximate the steady-state distribution for the conditions after the pulse.

both models is excellent. Apparently, the changing shape of the collapsing distribution at those sizes is primarily caused by the size dependence of the growth rate, and not by diffusion in size space. This is somewhat surprising for droplets near the critical radius $r_{1}^{*}$, where the drift flux $\dot{r} F$ in the Fokker-Planck equation [Eq. (13)] is small and the diffusion flux is dominant. Let us analyze the behavior at the critical size in detail, assuming that the Fokker-Planck equation is a good approximation of the kinetic equation. The drift flux is zero, but the GDE agrees with the kinetic equation, so the diffusion term must be negligible. From the Fokker-Planck equation, we find that the diffusion term is negligible compared to the drift term if

$$
\left|\frac{\partial D}{\partial r} \frac{\partial F}{\partial r}+D \frac{\partial^{2} F}{\partial r^{2}}\right| \ll\left|F \frac{\partial \dot{r}}{\partial r}\right| .
$$

This condition is satisfied for relatively large values of $F$ with small enough first and second derivatives. Therefore, although the drift $f u x \dot{r} F$ is zero at the critical radius, the drift term $\partial(\dot{r} F) / \partial r$ can be dominant compared to the diffusion term.

In nucleation pulse experiments, the number of droplets that are larger than a certain radius is detected. This smallest detectable radius is usually much larger than the critical size. Therefore, the large difference between the GDE and the kinetic equation at small sizes has no influence on the predicted number of detected droplets. The only remaining difference is that of the front of the distribution. As we have shown in Sec. IV A, this difference can be minimized if a suitable initial radius is chosen in the GDE. The predicted number of detectable droplets is then the same in both models.

Let us consider how the nucleation pulse is used experimentally to determine nucleation rates. It is assumed that the nucleation rate is constant in time and all nuclei are eventually detected, and the experimental nucleation rate is computed from ${ }^{25}$

$$
J_{\text {expt }}=\frac{\rho_{\text {expt }}}{\Delta t},
$$

where $\rho_{\text {expt }}$ is the number density of detected droplets and $\Delta t$ is the pulse duration. The underlying assumption is that

$$
\rho_{\text {expt }} \approx J \Delta t,
$$

which is not exactly true because it takes a certain time to establish the steady-state $J$ and to stop the nucleation after the end of the pulse. Furthermore, at the end of the pulse $r^{*}$ increases, so some droplets become subcritical, then evaporate, and are not detected. The kinetic model allows us to test the accuracy of the approximation in Eq. (34), by computing the ratio of detected droplets, defined here as

$$
d \equiv \frac{\rho_{\text {kin }}}{J \Delta t},
$$

where $\rho_{\text {kin }}$ is the number density of droplets that reach a detectable size in the kinetic model. As long as the detectable size is much larger than the critical size, $d$ does not depend on the detectable size. The ratio $d$ then depends on five parameters: the pulse conditions $S_{0}$ and $\Theta_{0}$, the conditions after the pulse $S_{1}$ and $\Theta_{1}$, and the dimensionless pulse duration $\Delta \tau$. The number of parameters is too large to compute the ratio $d$ for all possible conditions. Therefore, we have evaluated it for two sets of $S$ and $\Theta$ values, as a function of $\Delta \tau$, shown in Fig. 6.

For short pulses the detected ratio is small because most droplets become subcritical after the pulse and evaporate. For long pulses, the number of evaporating droplets and the transient behavior of $J$ become relatively unimportant. However, care must be taken that the pulse is not made too long because depletion cannot be ignored for long pulses. The effects of depletion are not included in our current model, so Fig. 6 does not show the errors made for long pulse dura- 


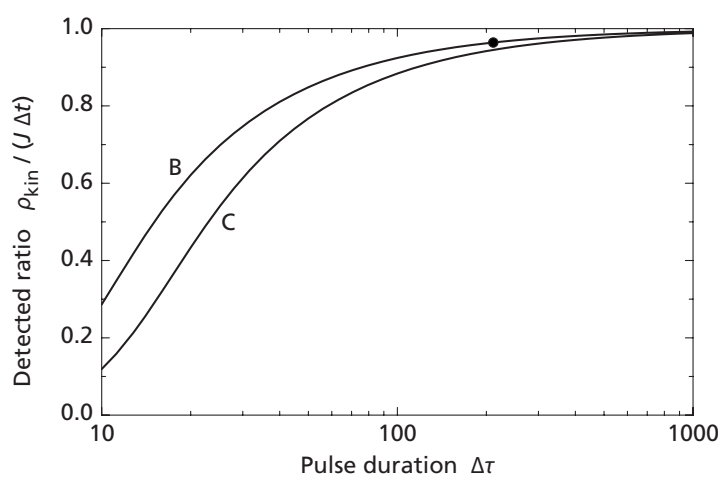

FIG. 6. Ratio of the detected number density of droplets and the assumed number density in the nucleation pulse method $J \Delta t$, as a function of the dimensionless pulse duration. Line B corresponds to typical pulse conditions from experiments in our group (Ref. 23). The dot represents the pulse duration $\Delta \tau=211$ that was experimentally used for these conditions. Line $\mathrm{C}$ represents a typical pulse condition from the experiments of Wölk and Strey (Ref. 22), with experimental pulse duration $\Delta \tau \approx 1.4 \times 10^{4}$. Conditions are listed in Table I.

tions. Conversely, depletion is negligible for short pulses, so Fig. 6 can be used to obtain a lower limit for the pulse duration.

\section{v. CONCLUSION}

We have addressed the problem of the formation of droplets in a supersaturated vapor, both with a discrete kinetic equation and a continuous general dynamic equation. The relationship between the equations can be easily understood by writing the kinetic equation as a Fokker-Planck equation in continuous radius coordinates. Neglecting the diffusion term in this equation immediately leads to the GDE.

The advantage of the kinetic equation is that it correctly describes nucleation and growth simultaneously. This is not trivial for the GDE since it shows a singular behavior near the critical radius. It is therefore necessary to change the GDE such that new droplets are introduced at a radius that is slightly larger than the critical radius. By adjusting this initial radius, selected differences between the GDE and the kinetic equation can be minimized. As an example, we have shown the initial radius that is required for agreement of the total number density of large droplets.

We have compared the kinetic equation and the GDE for constant temperature and supersaturation and in a nucleation pulse. Three radius ranges can be distinguished. First, the shape of the front is sharp in the GDE size distribution and smooth in the kinetic one, illustrating the absence in the GDE of diffusion in size space. Second, for droplet sizes between the critical region and the front region, the GDE agrees well with the kinetic equation. If nucleation is unim- portant compared to the existing droplet concentration, the GDE is also accurate in the critical region. Finally, at the lower end of the size range, between monomers and the critical size, large differences between the models are inevitable. The kinetic distribution is high in this size range because of the high concentration of monomers, whereas the GDE distribution approaches zero for small radii.

The application of the kinetic model to a nucleation pulse enabled us to assess the accuracy of the nucleation pulse method as it is experimentally used. It was found that short pulses lead to an evaporation of a considerable part of the droplets that are formed, which decreases the accuracy of the determined nucleation rate.

A limitation of this study is that the models have not been evaluated for continuously changing conditions, such as the flow in a nozzle. In such a situation, the agreement between the models could be different.

${ }^{1}$ T. W. Peterson, F. Gelbard, and J. H. Seinfeld, J. Colloid Interface Sci. 63, 426 (1978).

${ }^{2}$ J. H. Seinfeld and S. N. Pandis, Atmospheric Chemistry and Physics: From Air Pollution to Climate Change (Wiley, New York, 1998).

${ }^{3}$ M. M. R. Williams and S. K. Loyalka, Aerosol Science: Theory and Practice (Pergamon, Oxford, 1991).

${ }^{4}$ Z. Kožíšek, K. Sato, P. Demo, and A. M. Sveshnikov, J. Chem. Phys. 120, 6660 (2004).

${ }^{5}$ E. N. Chesnokov and L. N. Krasnoperov, J. Chem. Phys. 126, 144504 (2007).

${ }^{6}$ W. G. Courtney, J. Chem. Phys. 36, 2009 (1962).

${ }^{7}$ F. F. Abraham, J. Chem. Phys. 51, 1632 (1969).

${ }^{8}$ F. Gelbard and J. H. Seinfeld, J. Colloid Interface Sci. 68, 363 (1979).

${ }^{9}$ R. Hagmeijer, R. H. A. IJzermans, and F. Put, J. Fluid Mech. 17, 056101 (2005).

${ }^{10}$ F. F. Abraham, Homogeneous Nucleation Theory: The Pretransition Theory of Vapor Condensation (Academic, New York, 1974).

${ }^{11}$ D. Kashchiev, Nucleation: Basic Theory with Applications (ButterworthHeinemann, Oxford, 2000).

${ }^{12}$ W. G. Courtney, J. Chem. Phys. 35, 2249 (1961).

${ }^{13}$ G. Wilemski, J. Chem. Phys. 103, 1119 (1995).

${ }^{14}$ J. L. Katz and H. Wiedersich, J. Colloid Interface Sci. 61, 351 (1977).

${ }^{15} \mathrm{H}$. Risken, The Fokker-Planck Equation: Methods of Solution and Applications, 2nd ed. (Springer, New York, 1989).

${ }^{16}$ J. R. Brock, Aerosol Sci. Technol. 2, 109 (1982).

${ }^{17}$ R. Hagmeijer, Phys. Fluids 16, 176 (2004).

${ }^{18}$ S. K. Loyalka and J. W. Park, J. Colloid Interface Sci. 125, 712 (1988).

${ }^{19}$ F. Gelbard, Y. Tambour, and J. H. Seinfeld, J. Colloid Interface Sci. 76, 541 (1980).

${ }^{20}$ J. D. Landgrebe and S. E. Pratsinis, J. Colloid Interface Sci. 139, 63 (1990).

${ }^{21}$ W. H. Press, S. A. Teukolsky, W. T. Vetterling, and B. P. Flannery, Numerical Recipes in C: The Art of Scientific Computing, 2nd ed. (Cambridge University Press, Cambridge, 1992).

${ }^{22}$ J. Wölk and R. Strey, J. Phys. Chem. B 105, 11683 (2001).

${ }^{23}$ V. Holten, D. G. Labetski, and M. E. H. van Dongen, J. Chem. Phys. 123, 104505 (2005).

${ }^{24}$ T. E. Ramabhadran, T. W. Peterson, and J. H. Seinfeld, AIChE J. 22, 840 (1976).

${ }^{25}$ R. Strey, P. E. Wagner, and Y. Viisanen, J. Phys. Chem. 98, 7748 (1994). 